\title{
Tamoxifen for Breast Cancer
}

\author{
Karn A, ${ }^{1}$ Jha AK, ${ }^{1}$ Shrestha S, ${ }^{1}$ Acharya B, ${ }^{1}$ Poudel S, ${ }^{1}$ Bhandari RB ${ }^{1}$ \\ 'Department of Radiation Oncology, B P Koirala Memorial Cancer Hospital, Bharatpur, Nepal.
}

\section{ABSTRACT}

Breast cancer is one of the common cancers. Hormonal therapy along with surgery, chemotherapy, radiotherapy and targeted therapy are vital modalities for the management of breast cancer. Tamoxifen has been the most widely used hormonal therapy for more than two decades. In this article we review the benefits, dose, duration and timing of Tamoxifen therapy in patients with breast cancer.

Key Words: breast cancer, hormonal therapy, tamoxifen

\section{INTRODUCTION}

Breast cancer ranks second as a cause of cancer death in women (after lung cancer). The 5-year relative survival for early breast cancer (EBC), locally advanced breast cancer ( $\mathrm{LABC}$ ) and metastatic breast cancer (MBC) are $98 \%, 84 \%$ and $27 \%$ respectively. ${ }^{1}$ Treatment modalities in breast cancer include surgery, radiation therapy (RT), chemotherapy (CT), hormonal therapy $(\mathrm{HT})$ and targeted therapy often in combination.

A number of factors influence the prognosis and the treatment decisions in the management of breast cancer. They are age, estrogen receptor (ER) / progesterone receptor (PR) status, HER 2 gene status, menopausal status, TNM stage, lympho-vascular space invasion and histologic grade. ${ }^{2}$ Adjuvant systemic therapy decisions are made mainly on (i) HER 2 gene amplification or overexpression (ii) estimated hormonal responsiveness of tumor and (iii) risk of relapse. About $20 \%$ of all breast cancers occur in women below 50 years, and $60 \%$ of these are ER positive, compared with $80 \%$ being ER positive in women above 50 years. ${ }^{3}$

Patients with ER positive tumors tend to have more indolent course and metastasize preferentially to soft tissues and bone. They are more often well differentiated and are associated with other favorable prognostic

\author{
Correspondence: \\ Dr. Ambuj Karn \\ Department of Radiation Oncology \\ BP Koirala Memorial Cancer Hospital \\ Bharatpur, Nepal \\ Email: sabiamb@yahoo.co.in \\ Phone: 9845176694
}


characteristics. Those with ER negative tumors relapse earlier and mostly metastasize to liver, lung, and brain. Hormonal therapies include selective estrogen receptor modulators (SERM), ovarian ablation (OA), luteinizing hormone-releasing hormone agonist (LHRHA), progestins, androgens, estrogens, corticosteroids, antiandrogens, and aromatase inhibitors (Als).

\section{Hormonal responsiveness}

Hormone responsive breast cancers have clear or high level of ER and/or PR expression. Patients with total lack of both ER and PR expression are hormone nonresponsive. Though the value of HT is influenced not only by the extent of receptor expression (tumor with greater percentages of positive cells derive greater benefits) ${ }^{4}$ but also by the quality of receptor expression (tumor with both ER and PR positive cells derive greater benefits from $\mathrm{HT}$ than single receptor positive cells), ${ }^{5}$ tumors with even only $1 \%$ or few cells staining positive for ER or PR, derive benefit from HT by reducing risk of metastasis. ${ }^{4,6,7}$ For these reasons, it is most appropriate that ER/PR expression should be reported as percentage of cells staining for each receptor. It seems to be irrational description of labeling a tumor ER/PR negative when less than 10\% cells stain positive for receptors.

\section{Tamoxifen}

Tamoxifen, a SERM, is an antiestrogen that binds competitively to ER. ${ }^{8}$ It blocks angiogenesis, lowers IGF1 and TGF production; and induces production of TGFcalmodulin and protein kinase $\mathrm{C}$. It also increases natural killer cell activity. In addition to antagonist effects, agonist effects include tumor flare, preservation or enhancement of bone mineral density, decrease in cholesterol level, and additional modifications of serum lipid profile.

\section{Tamoxifen and Hormonal Receptors}

Tamoxifen has proven to be the most effective HT for receptor positives. Receptor positive tumors should be considered for adjuvant HT regardless of status of age, menopause, Her 2 gene, axillary lymph node (ALN) and adjuvant $\mathrm{CT}{ }^{6}$

When accurately measured, not only ER, but also PR status best predict response to tamoxifen. Review of two large databases encompassing more than 15,000 EBC patients (both pre and post menopausal and none receiving adjuvant $C T$ ) confirmed value of $P R$ in addition to ER in determining potential benefits of tamoxifen. ${ }^{5}$ Tamoxifen significantly decreased risk of mortality in PR positives compared to negatives. Similar results have also been noted for MBC.
Both ER and PR clearly have prognostic value, although their ability to discriminate between low and high risk is quite limited. In patients with negative ALNs, ER status is weak discriminant between high and low risk. Differences in outcome between ER negative and ER positive, node negative are of insufficient magnitude to base treatment decisions on receptor data alone. Receptor data may be of greater value when combined with other prognostic factors. ${ }^{9}$

Only hormone receptor positive patients should receive tamoxifen. ${ }^{6,10}$ Hormone nonresponsives derive greater benefit from CT and should not receive HT as they do not respond to or derive benefit from HT rather may be detrimental, ${ }^{6}$ adversely affecting relapse free and overall survival. ${ }^{11,12}$

HT also has a role as neo-adjuvant therapy, particularly when diagnostic biopsy results confirm receptor expression. $^{13,14}$

\section{Dose and duration of Tamoxifen administration}

Tamoxifen and its active metabolites have prolonged serum half-life (seven days) after reaching steady-state levels. ${ }^{15}$ Missing occasional dose does not alter serum levels dramatically and no extra dose is required for missed one.

Higher dosages have not been associated with improved outcome and lower dosages have not been adequately tested. Once daily $20 \mathrm{mg}$ of tamoxifen appears optimal and sufficient. Five years of tamoxifen is more effective than 1 or 2 years. ${ }^{16}$ Five years of tamoxifen reduces annual recurrence risk by $39 \%$ and mortality risk by $31 \%$ which translates into 15 -year absolute recurrence risk reduction of $12 \%$ and mortality risk reduction of $9 \%$ in ER or PR positives. ${ }^{17}$ Benefits are similar in both pre and post menopausals as well as in older and MBC. It was speculated that if it was given for only five years, tumor growth might merely be delayed for short time but tumor will subsequently appear after discontinuation. NSABP B-14 (a randomized, double-blind, placebo controlled trial to evaluate benefit of tamoxifen in patients with node negative ER positive tumors) did not support the speculation of regrowth after discontinuation. ${ }^{18}$ Overall disease free survival (DFS) was highly significant for tamoxifen ( $p<0.0001$ ), for both below and above 50 years ( $p=0.0001$ for both). Also, tamoxifen treated patients had fewer locoregional and distant failures. Ipsilateral and contralateral breast tumor recurrences were reduced by $58 \%$ and $40 \%$ respectively in tamoxifen arm. A significant survival advantage was also evident ( $p=0.02$ ). Tamoxifen-treated patients, who remained disease free after five years, were again randomized to 
continue tamoxifen or placebo for another five years to obtain information on appropriate duration of treatment. After four years, patients reassigned to placebo had a significantly better DFS ( $p=0.003$ ) and marginal overall survival benefit ( $p=0.08$ ) compared to those who continued tamoxifen. Results remained unchanged after seven years of follow-up. Reduction in incidence of contralateral breast cancer observed with five years of tamoxifen remained almost same through 10 years of follow-up with $37 \%$ decrease at that time. Similar results were obtained from a Scottish trial. ${ }^{19}$ Though there are no compelling evidences ${ }^{20-22}$ that prolonging tamoxifen for more than five years will provide greater benefit, controversy is still going on whether longer than five years of tamoxifen may be more effective. Two ongoing large randomized trials, Adjuvant Tamoxifen-Longer Against Shorter (ATLAS) trial and Adjuvant Tamoxifen Treatment Offer More? (aTTom) trial currently in progress, should help resolve this issue. At present, five years of duration is data driven and reasonable.

\section{Tamoxifen in HER 2 gene positive tumors}

There are conflicting results whether HER 2 gene positive breast cancers are resistant to tamoxifen when they are hormone receptor positive too. ${ }^{23-26}$ Additional trials are needed to clarify this issue. At present, no justifications for withholding tamoxifen in such situations are present. National Comprehensive Cancer Network (NCCN) recommends for $\mathrm{HT}$ in such situation. ${ }^{27}$

\section{Tamoxifen with Chemotherapy}

Tamoxifen combined with CT is the most effective adjuvant therapy in receptor positive $\mathrm{EBC}$ and $\mathrm{LABC}$. $^{6,14,28}$ NSABP B-16 was carried out to determine whether tamoxifen used in conjunction with each of two different CT regimens is more effective in prolonging DFS and overall survival (OS) than is tamoxifen alone in tamoxifenresponsives. ${ }^{29}$ Patients were randomized to receive (1) tamoxifen alone, (2) Adriamycin and cyclophosphamide plus tamoxifen; or (3) PFT (L-Pam, 5-FU, and tamoxifen), which was subsequently modified by adding Adriamycin (PAFT). Through seven years of follow-up, there was significantly better DFS for adjuvant CT treated patients than for those receiving tamoxifen alone ( $p=0.0009$ ). Significant OS advantage ( $p=0.02$ ) was also observed. Both DFS and OS of PAFT treated patients were significantly better ( $p=0.003$ and $p=0.05)$ than in those treated by tamoxifen alone. The study concluded better outcome from use of adjuvant tamoxifen and short course AC therapy than from tamoxifen alone in node positive patients of age 50 or above. Obviously, patients with poor performance status would be prohibited from toxicity of CT and are best treated by tamoxifen alone.
Earlier laboratory and clinical data suggested unfavorable, might be antagonistic effects in concurrent chemohormonal therapy in some subgroups. ${ }^{30-32}$ Even increased risk of thromboembolic complications were observed when tamoxifen and CT were administered simultaneously particularly in women above 50 years. ${ }^{33-35}$

Current practice is to administer tamoxifen sequentially after chemotherapy rather than concurrently based on the Southwest Oncology Group Intergroup Trial S8814 (INT-0100), which reported a modest improvement in outcome with this approach. In this study, 1,477 receptor and node positive post menopausal women were randomized to receive either tamoxifen alone, concurrent tamoxifen and CAF, or sequential CAF followed by tamoxifen. At a median follow up of 8.5 years, 8 -year DFS estimates were $67 \%$ for sequential CAF followed by tamoxifen and $62 \%$ for concurrent CAF plus tamoxifen $(p=0.045)$, showing slight worse DFS and OS (adjusted $\mathrm{HR}, 1.2$ and 1.12 , respectively) for concurrent arm. ${ }^{36}$

These results suggest that tamoxifen should be given after completion of CT (sequential administration) and not along with CT (concurrent administration) in patients who receive both $\mathrm{CT}$ and tamoxifen.

\section{Tamoxifen in old age and MBC}

Results from Early Breast Cancer Trialists' Collaborative Group (EBCTCG) shows that, in women 70 years or above with ER positive, tamoxifen yields significant improvement in both DFS and $\mathrm{OS}^{6}$. Anastrozole is an alternative.37

Tamoxifen produces tumor regression in $50-60 \%$ of $\mathrm{MBC}$ patients with ER positive. ${ }^{38}$ Responses last 12 months, on average. In addition, another 20 to $30 \%$ of women have stable disease (SD) for more than six months and, in some cases, even longer. Due to excellent tolerance, tamoxifen has become front-line HT of choice for untreated $\mathrm{MBC}$. Tamoxifen is at least as effective as other HT for both pre and post menopausal. ${ }^{39}$ Tamoxifen is also active as second line HT in patients responding to, and then progressing after, ablative surgery, and progestins. Premenopausal that had not received prior tamoxifen or had received but developed metastases, may still respond later to tamoxifen rechallenge. In those patients, tamoxifen is likely to be almost as effective as when used in $\mathrm{HT}$-naive patients especially if intervening tamoxifenfree interval is long. ${ }^{40}$ In premenopausal, similar efficacy for tamoxifen and $O A$ has been found. ${ }^{41}$ Choice of tamoxifen or OA as initial HT should be based on patient and physician preference. Otherwise, tamoxifen with $O A$ or Als after or concomitantly with OA should be considered. In postmenopausal, HT should be individualized according to patient's safety profile, and tamoxifen 
remains an acceptable first line therapy in selected cases. Als are superior to progestins and equal if not superior to tamoxifen as initial treatment. ${ }^{42}$ When compared to tamoxifen as first line HT, Als have shown improved overall responses and time to progression, but no differences in survival.

\section{Tamoxifen in Carcinoma in situ}

Apart from invasive cancer, tamoxifen is also indicated in carcinoma in situ (CIS). NSABP P-1 trial $^{43}$ showed tamoxifen led to $50 \%$ fewer noninvasive and invasive tumors (both ductal CIS and lobular CIS) than placebo. Invasive breast cancer reduction noted was $69 \%$ in ER positive. No such reduction observed in ER negative. NSABP B-24 was another trial observing decreased rate of invasive carcinoma, especially in ipsilateral breast by radiotherapy and tamoxifen over $\mathrm{RT}$ and placebo. ${ }^{44}$ Incidence of all cancer events at five years decreased from $13.2 \%$ in placebo to $8.2 \%$ in tamoxifen. Cumulative incidence of all cancer reduced from $7.2 \%$ in placebo to $4.1 \%$ in tamoxifen. Tamoxifen resulted in $38 \%$ reduction in ipsilateral tumor in below 50 years and $22 \%$ reduction in above 50 years. Benefits of tamoxifen in DCIS were restricted to receptor positive. Previously NSABP B-17 had similar results. ${ }^{45}$ From above, it is clear that tamoxifen provides significant benefit in risk reduction for all categories: age, tumor size, node status and family history.

\section{Adverse effects of Tamoxifen}

Nausea, hot flashes and vaginal discharges are common. Posterior subcapsular opacities have been found frequently but macular degeneration or vision threatening toxicities have not been found. Agonist effects like tumor flare and thromboembolic events (stroke, pulmonary embolism, and deep vein thrombosis; less than $1 \%$ each) may occur. In about $10 \%$ patients, there may be an initial, shortlasting tumor flare, starting within first week or two of administration. This may be manifested as increased bone pain, development of hypercalcemia, or, occasionally, even a slight increase in tumor dimensions. Flare usually predicts response to therapy, and close follow-up with active symptomatic support is appropriate. If tumor flare symptoms appear late (after more than three weeks), they represent progression of disease and should prompt discontinuation of tamoxifen and change in therapy. Serum estrogen level rises dramatically in some premenopausals, but this effect does not mediate tamoxifen resistance. Tamoxifen in premenopausal is not associated with increased risk of venous thromboembolism. Though endometrial hyperplasia and ovarian cysts have been seen not infrequently, no increased risk of second tumors except endometrial malignancies and few uterine sarcomas are documented
(NSABP B-14). ${ }^{18}$ In postmenopausal above 50 years, five years of tamoxifen was associated with a $1 \%$ risk of second malignancy. Although risk increases after tamoxifen, net benefit vis-à-vis breast cancer greatly outweighs risk. Endometrial cancer occurring after tamoxifen does not appear to be of different type or of worse prognosis than those not receiving tamoxifen. Women taking tamoxifen should undergo annual gynecologic evaluation. Leucopenia and thrombocytopenia are rare. There are conflicting results regarding depression, sexual dysfunction, fluid retention and weight gain. Other hormonal agents

In premenopausal, Tamoxifen and / or OA is indicated if tumor is responsive. Tamoxifen and LHRH is also an option but $\mathrm{Al}$ alone must be avoided as no evidence of efficacy is available in premenopausal. In postmenopausal, HT should include Al at some point in time. It is as yet undetermined whether Al should be used upfront and for which exact time period. Alternatively, tamoxifen is still an option, with a planned switch to an Al after 2-3 or five years. Als have shown to increase DFS when compared with tamoxifen. This has been shown for anastrozole (1 mg/day for five years) and for letrozole ( $2.5 \mathrm{mg} /$ day for five years) given upfront, for exemestane (25 mg/day) and for anastrozole (1 mg/day) given after 2-3 years of tamoxifen, and for letrozole $(2.5 \mathrm{mg} /$ day $)$ and anastrozole compared with placebo given after five years of tamoxifen.

MA-17 trial compared letrozole with placebo in 5000 post-menopausal with hormone receptor positive who had received five years of tamoxifen. This trial showed decrease in breast cancer related events (new breast cancers, local-regional recurrence, and metastases) after a median follow-up of 28 months. ${ }^{46}$

ATAC (Arimidex, Tamoxifen, Alone or in Combination) trial randomly assigned 9366 receptor positive postmenopausal to receive anastrozole (arimidex), tamoxifen, alone or combination of both. ${ }^{37}$ Anastrozole had significantly decreased hazard ratio $10.82 ; 95 \%$ confidence interval, 0.70 to 0.96 ) for breast cancer related event (new breast cancer, local recurrence, or metastases) compared to patients taking tamoxifen or combination. Anastrozole was associated with $17 \%$ decrease in relative risk of recurrences. Relapse-free survival at four years was $89 \%$ and $86 \%$, respectively, for anastrozole alone and for tamoxifen or combination. Absolute difference in frequency of metastases at four years was $1 \%$ for these two groups, though no significant differences in OS was been found.

Intergroup Exemestane Trial randomly assigned 5000 women who had received tamoxifen for 2-3 years to receive 2-3 years more either tamoxifen or exemestane. At a median follow-up of 31 months, patients receiving 
exemestane had a significant decrease in breast cancer related relapse compared to those continuing on tamoxifen (hazard ratio, 0.68; $\mathrm{P}<.001) .{ }^{47}$ No survival differences were noted.

\section{Hormone withdrawal}

Hormonal withdrawal is seen with high-dose estrogen, tamoxifen, androgens and progestins. Suggested mechanism is that, after long-term suppression of cancer cell lines by HT, some cells become dependent on (or stimulated by) continued exposure to it. ${ }^{48}$ At progression if $\mathrm{HT}$ is removed, secondary inhibition of tumor cells can be observed. For indolent metastasis, especially who have no symptoms at time of secondary progression after intervening response to $\mathrm{HT}$ (e.g., relapsing while taking tamoxifen, with disease-free interval of two years or more) period of 2-3 months of observation (drug holiday) to detect withdrawal response might be appropriate.

\section{CONCLUSIONS}

Not only ER but also PR expression along with percentage of cells staining for receptor is mandatory in the management of breast cancer. Responsive tumors should be considered for adjuvant HT regardless of age, menopause, HER 2 gene, axillary lymph node and chemotherapy. Non responsive tumors should not receive HT empirically. Tamoxifen has proven to be the most effective HT for receptor positives. HER 2 positive tumors also need tamoxifen if they are receptor responsive too. Tamoxifen should be administered after completion of chemotherapy. Missing occasional dose does not require additional dose. Tamoxifen (20 mg / day) for five years is data driven and reasonable.

\section{REFERENCES}

1. American Cancer Society. Cancer Facts \& Figures. Atlanta: American Cancer Society; 2008.

2. Henderson C, Patek A. Relationship between prognostic and predictive factors in management of breast ca. Breast Cancer Res Treat. 1998;52:261.

3. Anderson WF, Chatterjee N, Ershler WB, et al. Estrogen receptor breast cancer phenotypes in the Surveillance, Epidemiology, and End Results database. Breast Cancer Res Treat. 2002;76:2736.

4. Harvey JM, Clark GM, Osborne CK, Allred DC. Estrogen receptor status by IHC is superior to the ligand-binding assay for predicting response to adjuvant endocrine therapy in breast cancer. J Clin Oncol. 1999;17:1474.

5. Bardou VJ, Arpino G, Elledge RM, Osborne CK, Clark GM. Progesterone receptor status significantly improves outcome prediction over estrogen receptor status alone for adjuvant endocrine therapy in two large breast cancer databases. J Clin Oncol. 2003;21:1973.

6. Early Breast Cancer Trialists' Collaborative Group. Tamoxifen for early breast cancer: an overview of the randomized trials. Lancet. 1998;351:1451-67.

7. Elledge RM, Green S, Pugh R, et al. ER and PR, by ligandbinding assay compared with ER, PR and pS2, by immunohistochemistry in predicting response to tamoxifen in metastatic breast cancer: a Southwest Oncology Group Study. Int J Cancer. 2000;89:111.

8. Rochefort $\mathrm{H}$. Mechanism of action of high affinity antiestrogens. An overview. Am J Clin Oncol. 1991;14S2:1-4.

9. Sundquist M, Thorstenson S, Brudin L, et al. Applying the Nottingham Prognostic Index to a Swedish breast cancer population. South East Swedish Breast Cancer Study Group. Breast Cancer Res Treat. 1999;53:1-8.
10. Fisher B, Anderson S, Tan-Chiu E, et al. Tamoxifen and chemotherapy for axillary node-negative, estrogen receptornegative breast cancer. Findings from NSABP B-23. J Clin Oncol. 2001;19:931-42.

11. Fisher B, Redmond C, Brown A, et al. Treatment of primary breast cancer with chemotherapy and tamoxifen. N Engl J Med. 1981;305:1-6.

12. Hutchins L, Green S, Ravdin P, et al. CMF versus CAF with and without tamoxifen in high-risk node-negative breast cancer patients and a natural history follow-up study in low-risk nodenegative patients. First results of inter-group trial INT 0102. Proc Am Soc Clin Oncol. 1998;17:1a.

13. Dixon J, Love C, Bellamy C, et al. Letrozole as primary medical therapy for locally advanced and large operable breast cancer. Breast Cancer Res Treat. 2001;66:191.

14. Rubens R, Bartelink H, Engelsman E, et al. Locally advanced breast cancer: the contribution of cytotoxic and endocrine treatment to radiotherapy. EORTC Breast Cancer Cooperative Group trial 10792. Eur J Cancer Clin Oncol. 1989;25:667.

15. Jordan VC. Metabolites of tamoxifen in animals and man. Identification, pharmacology, and significance. Breast Cancer Res Treat. 1982;2:123-38.

16. Swedish Breast Cancer Cooperative Group. Randomized trial of two versus five years of adjuvant tamoxifen for postmenopausal early stage breast cancer. Swedish Breast Cancer Cooperative Group. J Nat Cancer Inst. 1996;88:1543-49.

17. Early Breast Cancer Trialists' Collaborative Group. Effects of chemotherapy and hormonal therapy for early breast cancer on recurrence and 15-year survival: An overview of the randomized trials. Lancet. 2005;365:1687-717.

18. Fisher B, Dignam J, Bryant J, et al. Five versus more than 5 years of tamoxifen therapy for breast cancer patients with negative lymph nodes and ER-positive tumors. J Natl Cancer Inst. 
1996;88:1529-42.

19. Stewart HJ, Forrest AP, Everington D, et al. Randomized comparison of 5 years of adjuvant tamoxifen with continuous therapy for operable breast cancer. Scottish Cancer Trials Breast Group. Br J Cancer. 1996;74:297-9.

20. Current Trials Working Party of the Cancer Research Campaign Breast Cancer Trials Group. Preliminary results from the cancer research campaign trial evaluating tamoxifen duration in women aged fifty years or older with breast cancer. Current Trials. J Nat Cancer Inst. 1996;88:1834-9.

21. Tormey DC, Gray R, Falkson HC. Postchemotherapy adjuvant tamoxifen therapy beyond five years in patients with lymph node-positive breast cancer. Eastern Cooperative Oncology Group. J Nat Cancer Inst. 1996;88:1828-33.

22. Stewart HJ, Scottish Breast Group. Adjuvant tamoxifen duration in a randomized trial. Breast. 1995;4:256.

23. Thor A, Berry D, Budman D, et al. erbB-2, p53, and efficacy of adjuvant therapy in lymph node positive, breast cancer. J Natl Cancer Inst. 1998;90:1346-60.

24. Carlomagno C, Perrone F, Gallo C, et al. C-erb B2 overexpression decreases the benefit of adjuvant tamoxifen in early stage breast cancer without axillary lymph node metastases. J Clin Oncol. 1996;14:2702-8.

25. Elledge R, Green S, Ciocca D, et al. HER-2neu expression does not predict response to tamoxifen in ER positive metastatic breast cancer. Breast Cancer Res Treat. 1996;41:289.

26. Pietras RJ, Arboleda J, Reese DM, et al. HER-2 tyrosine kinase pathway targets estrogen receptor and promotes hormoneindependent growth in human breast cancer cells. Oncogene. 1995;10:2435-46.

27. NCCN Clinical Practice Guidelines in Oncology. Breast Cancer. V.I.2009. National Comprehensive Cancer Network. [Online]. 2009[Cited 2009 Nov 12]; Available from: .

28. Early Breast Cancer Trialists' Collaborative Group. Effects of adjuvant tamoxifen and of cytotoxic therapy on mortality in early breast cancer. An overview of 61 randomtality in early breast cancer. An overview of 61 randomized trials among 28,896 women. N Engl J Med. 1988;319:1681.

29. Fisher B, Redmond C, Legault-Poisson S, et al. Postoperative chemotherapy and tamoxifen compared with tamoxifen alone in the treatment of positive-node breast cancer patients aged 50 years and older with tumors responsive to tamoxifen. Results from the National Surgical Adjuvant Breast and Bowel Project B-16. J Clin Oncol. 1990;8:1005-18.

30. Hug V, Thames H, Clark J. Chemotherapy and hormonal therapy in combination. J Clin Oncol. 1988;6:173-7.

31. Osborne C, Kitten L, Arteaga C. Antagonism of chemotherapy induced cytotoxicity for human breast cancer cells by antiestrogens. J Clin Oncol. 1989;7:710-7.

32. Fisher B. Treatment of primary breast cancer with L-PAM/5FU and tamoxifen. An interim report. Breast Cancer Res Treat. 1983;3 Suppl:S7-17.490.

33. Levine MN, Gent M, Hirsh J, et al. The thrombogenic effect of anticancer drug therapy in women with stage II breast ca. N Engl J Med. 1988;318:404-7.

34. Pritchard K, Paterson A, Paul N, et al. Increased thromboembolic complications with concurrent tamoxifen and chemotherapy in a randomized trial of adjuvant therapy for women with breast cancer. NCIC CTG. Breast Cancer Site Group. J Clin Oncol. 1996;14:2731-7.

35. Pritchard K, Paterson A, Fine S, et al. Randomized trial of cyclophosphamide, methotrexate, and fluorouracil chemotherapy added to tamoxifen as adjuvant therapy in postmenopausal women with node positive estrogen and/or progesterone receptor positive breast cancer. A report of the NCIC CTG. Breast Cancer Site Group. J Clin Oncol. 1997;15:2302-11.

36. Albain K, Warlow W, O`Malley F, et al. Concurrent (CAFT) versus sequential (CAF-T) chemohormonal therapy versus $\mathrm{T}$ alone for postmenopausal node positive and/or PR positive breast cancer: mature outcomes and new biologic correlates on phase III intergroup trial 0100. San Antonio breast cancer symposium; 2004 Dec 8-11; San Antonio, TX: 2004.

37. ATAC Trialists Group. Anastrozole alone or in combination with tamoxifen versus tamoxifen alone for adjuvant treatment of postmenopausal women with early breast cancer: first results of ATAC randomized trial. Lancet. 2002;359:2131.

38. Muss H, Case L, Atkins J, et al. Tamoxifen versus high-dose oral medroxyprog- esterone acetate as initial endocrine therapy for patients with metastatic breast cancer. A Piedmont Oncology Association study. J Clin Oncol. 1994;12:1630-8.

39. Osborne CK, Zhao H, Fuqua SA. Selective estrogen receptor modulators: structure, function, and clinical use. J Clin Oncol. 2000;18:3172.

40. Muss HB, Smith LR, Cooper MR. Tamoxifen rechallenge. Response to tamoxifen following relapse after adjuvant chemohormonal therapy for breast cancer. J Clin Oncol. 1987;5:1556-8.

41. Perez R, Alberola V, Calabresi F, et al. Comparison of selective aromatase inhibitor formestane with tamoxifen as first-line hormonal therapy in postmenopausal women with advanced breast cancer. Ann Oncol. 1994;5(7):19-24.

42. Smith IE, Dowsett M. Aromatase inhibitors in breast cancer. N Engl J Med. 2003;348:2431.

43. Fisher B, Costantino JP, Wickerham DL, et al. Tamoxifen for prevention of breast cancer: report of the National Surgical Adjuvant Breast and Bowel Project P-1 study. J Natl Cancer Inst. 1998;90:1371-88.

44. Allred DC, Bryant J, Land S, et al. Estrogen receptor expression as a predictive marker of the effectiveness of tamoxifen in the treatment of DCIS: findings from NSABP protocol B-24. Breast Cancer Res Treat. 2002;76:S36.

45. Fisher B, Dignam J, Wolmark N, et al. Lumpectomy and radiation therapy for the treatment of intraductal breast cancer. Findings from National Surgical Adjuvant Breast and Bowel Project B17. J Clin Oncol. 1998;16:441-52.

46. Goss PE, Ingle JN, Martino S, et al. A randomized trial of letrozole in postmenopausal women after five years of tamoxifen therapy for early-stage breast cancer. N Engl J Med. 2003;349:1793.

47. Coombes RC, Hall E, Gibson LJ, et al. A randomized trial of exemestane after two to three years of tamoxifen therapy in postmenopausal women with primary breast cancer. N Engl J Med. 2004;350:1081.

48. Gottardis M, Jordan V. Development of tamoxifen stimulated growth of mcf-7 tumors in athymic mice after long-term antiestrogen administration. Cancer Res. 1988;48:5183-7. 